\title{
Nonaxisymmetric high-aspect-ratio ellipsoids under shear: Lowest-order correction for finite aspect ratios
}

\author{
Feng Shi ${ }^{1,2}$ and Peter J. Mucha ${ }^{1}$ \\ ${ }^{1}$ Department of Mathematics, University of North Carolina, Chapel Hill, North Carolina 27599, USA \\ ${ }^{2}$ Computation Institute, University of Chicago, Chicago, IL 60637-2616, USA \\ (Received 21 August 2013; revised manuscript received 17 May 2014; published 8 July 2014)
}

\begin{abstract}
We extend the classic study of the motion of small ellipsoidal particles under shear, focusing on simplifications obtained by considerations of the extreme aspect ratios typical of rheoscopic particles (e.g., Kalliroscope). Specifically, we study conditions under which the long-time behavior of scalene (i.e., triaxial or nonaxisymmetric) ellipsoids are well approximated by a model that is low order in the appropriate aspect ratios. After enumerating and describing the generic long-time motions of such particles in the lowest-order model, we investigate corrections induced by the physically appropriate lowest-order correction to the base model, with special attention to a periodic wobbling motion special to scalene ellipsoids.
\end{abstract}

DOI: 10.1103/PhysRevE.90.013005

PACS number(s): 83.10.Pp, 83.80.Hj, 45.40.Bb, 45.10.Hj

\section{INTRODUCTION}

The motion of ellipsoidal particles immersed in flow is a long-studied area of fluid dynamics. Following Jeffery's seminal study [1] on the motion of axisymmetric ellipsoidal particles in a simple shear flow, there has been a significant amount of work devoted to experimental investigations of Jeffery's hypotheses [2,3] along with studies of the effects of inertia [4,5], particle-particle interactions [6] and Brownian motion [7-9].

The present work deviates from the above history by its focus on high-aspect-ratio nonaxisymmetric (also known as scalene or triaxial) ellipsoids in general shear flows, as motivated by rheoscopic fluids that utilize small reflective flakes and their collective reflection of light to visualize flow behaviors $[10,11]$. The resulting patterns of reflection are connected to the orientations of the particles. While particle orientations have been extensively studied in special flows both experimentally [12-16] and theoretically [17-20], there is no general analytical solution to the ordinary differential equations for the motion of a triaxial ellipsoid in threedimensional (3D) flows. As such, Gauthier et al. [21] modeled rheoscopic flow visualization by way of numerical solution for the motion of isolated scalene ellipsoids. Alternative approaches for modeling rheoscopic flow visualization based on (uncontrolled) simplifying assumptions were developed by Barth and Burns [22] and by Hecht et al. [23]. The latter model leverages the extreme aspect ratios of rheoscopic particles (e.g., Kalliroscope AQ1000 flakes [10] are $\left.30 \times 6 \times 0.07 \mu \mathrm{m}^{3}\right)$ to describe classes of generic long-time orientational behaviors of triaxial ellipsoids in locally linear flows, in terms of a base model that is zeroth-order in the particle aspect ratios.

Motivated by the ability of the Hecht et al. model to enumerate different classes of motion, we theoretically consider the lowest-order correction (that is, the first-order correction in the larger aspect ratio) in order to investigate the possible changes to particle orbits that are accessible from such a perturbative analysis. Even without a general analytical solution of the complete equations of motion for finite aspect ratios, this lowest-order perturbative analysis uncovers a triaxial ellipsoid wobbling motion that appears to have gone previously unrecognized.
Our starting point is the work of Bretherton [24] who investigated the orbit of a generally shaped particle in complicated shears and developed a method of eigenvalue analysis, which we will use in a specific manner to describe high-aspect-ratio scalene ellipsoids, extending the work of Hecht et al. [23]. We retain here the first correction due to finite values of the larger aspect ratio to obtain insight into the asymptotic trajectories of scalene particles. The introduction of this small perturbation can dramatically change the pattern of motion. For certain flows, the longest axis of an ellipsoid-aligning to a fixed direction in the zeroth-order base model-instead wobbles under perturbation around that same fixed vector. In Sec. II we briefly recall the analytical results for the motion of ellipsoidal particles and review the zeroth-order base model of Hecht et al. [23]. Section III introduces the theoretical framework for the lowest-order correction, with results from the perturbation analysis given in the remaining sections (IV, $\mathrm{V}$, and VI), including the heretofore unrecognized wobbling motion described in Sec. VI. Section VII discusses the domain of viability and the limitations of the lowest-order correction result with possible extensions of the perturbation analysis.

\section{DERIVATION OF THE MODEL}

We consider a 3D flow with velocity field $\mathbf{v}(\mathbf{x})=(u, v, w)$ and denote its gradient velocity tensor in Cartesian coordinates by $\mathbf{G}$, which can be decomposed into symmetric and antisymmetric components: the rate-of-deformation tensor $\mathbf{E}=\left(\mathbf{G}+\mathbf{G}^{T}\right) / 2$ and the vorticity tensor $\Omega=\left(\mathbf{G}-\mathbf{G}^{T}\right) / 2$. To characterize the motion of an ellipsoidal particle immersed in this flow, it is convenient to consider the right-handed orthonormal reference frame built along the instantaneous semiaxes of the ellipsoid, denoted $\hat{\mathbf{a}}, \hat{\mathbf{b}}$, and $\hat{\mathbf{c}}$, respectively. Then the orientation of the ellipsoid is described by the orientations of its three semiprincipal axes, with lengths $a$, $b$, and $c$.

Several simplifying assumptions are made throughout this paper. First, it is assumed that the size of the particle is small enough and its density is very close to that of the fluid so that its Reynolds number is very small. Second, all Brownian motion, inertial, and non-Newtonian effects are neglected. Third, we 
assume the instantaneous rotation of the particle responds quickly to the flow compared to the time rate of change of the local gradient tensor so the flow can be viewed as steady local to the particle. Finally, we assume the flow is locally linear on the length scale of the particle.

Following Jeffery [1] and Gauthier et al. [21], the orientation of the ellipsoid is elucidated by its angular velocity $\boldsymbol{\omega}$. The components of this vector expressed in the $(\hat{\mathbf{a}}, \hat{\mathbf{b}}, \hat{\mathbf{c}})$ frame of the particle, derived from a full Stokes flow analysis by Jeffery [1], are:

$$
\begin{aligned}
& \omega_{1}=\frac{b^{2} G_{32}-c^{2} G_{23}}{b^{2}+c^{2}}=\Omega_{32}+\frac{r_{1}^{2}-r_{2}^{2}}{r_{1}^{2}+r_{2}^{2}} E_{32}, \\
& \omega_{2}=\frac{c^{2} G_{13}-a^{2} G_{31}}{a^{2}+c^{2}}=\Omega_{13}+\frac{r_{2}^{2}-1}{r_{2}^{2}+1} E_{13}, \\
& \omega_{3}=\frac{a^{2} G_{21}-b^{2} G_{12}}{a^{2}+b^{2}}=\Omega_{21}+\frac{1-r_{1}^{2}}{1+r_{1}^{2}} E_{21},
\end{aligned}
$$

where $r_{1}=b / a$ and $r_{2}=c / a$ are aspect ratios. Bretherton [24] deftly treated the case of an axisymmetric $(b=c)$ particle, in which the orientation of the particle is fully characterized by the axis â and only one aspect ratio $r=b / a$ is present, by way of the equation

$$
\frac{d}{d t} \hat{\mathbf{a}}=\boldsymbol{\omega} \times \hat{\mathbf{a}}=\mathbf{P} \cdot \hat{\mathbf{a}}-P_{11} \hat{\mathbf{a}}
$$

where

$$
\mathbf{P}=\Omega+\frac{1-r^{2}}{1+r^{2}} \mathbf{E},
$$

Bretherton observed that the last term in Eq. (2), parallel to the direction of $\hat{\mathbf{a}}$, conserves the unit length of $\hat{\mathbf{a}}$ but does not affect its orientation. Therefore the long-time orientation of $\mathbf{a}$ is determined by the eigenvalues and eigenvectors of $\mathbf{P}$.

Hecht et al. [23] generalized Bretherton's idea to scalene particles with $r_{2} \ll r_{1} \ll 1$ as below. The full dynamics of the scalene ellipsoid obey

$$
\begin{aligned}
\frac{d}{d t} \hat{\mathbf{a}} & =\hat{\mathbf{b}}\left(G_{21}-\frac{2 r_{1}^{2}}{1+r_{1}^{2}} E_{21}\right)+\hat{\mathbf{c}}\left(G_{31}-\frac{2 r_{2}^{2}}{1+r_{2}^{2}} E_{31}\right), \\
\frac{d}{d t} \hat{\mathbf{c}} & =\hat{\mathbf{a}}\left(\frac{2 r_{2}^{2}}{1+r_{2}^{2}} E_{31}-G_{31}\right)+\hat{\mathbf{b}}\left(\frac{2 r_{2}^{2}}{r_{1}^{2}+r_{2}^{2}} E_{32}-G_{32}\right),
\end{aligned}
$$

obtained from, e.g., $\frac{d}{d t} \hat{\mathbf{a}}=\boldsymbol{\omega} \times \hat{\mathbf{a}}$ and Eq. (1). Neglecting $O\left(r^{2}\right)$ terms because of the extreme aspect ratios $\left(r_{2} \approx\right.$ $2 \times 10^{-3}$ and $r_{1} \approx 0.2$ for a Kalliroscope particle [10]) and applying Bretherton's treatment, Hecht et al. obtained the simplified dynamics

$$
\frac{d}{d t} \tilde{\mathbf{a}}=\mathbf{G} \cdot \tilde{\mathbf{a}}, \quad \frac{d}{d t} \tilde{\mathbf{c}}=-\mathbf{G}^{T} \cdot \tilde{\mathbf{c}},
$$

in the limit $r_{1} \rightarrow 0, r_{2} \rightarrow 0$, where $\tilde{\mathbf{a}}$ and $\tilde{\mathbf{c}}$ are vectors parallel to $\hat{\mathbf{a}}$ and $\hat{\mathbf{c}}$ respectively obtained by removing the unit-length constraints on $\hat{\mathbf{a}}$ and $\hat{\mathbf{c}}$ as in Bretherton [24]. The eigenvalue analysis of $\mathbf{G}$ and $-\mathbf{G}^{\mathbf{T}}$ then gives the orientation of the scalene ellipsoidal particle in its (statistically) steady state. Because the eigenvalues of $\mathbf{G}$ and $\mathbf{G}^{\mathbf{T}}$ are the same, and they must sum to zero for an incompressible flow, Hecht et al. identified three generic cases based on the signs and possible complex pairs of the eigenvalues. In contrast, the case where all real parts of eigenvalues are identically zero that corresponds to simple shear and Jeffery's orbits is nongeneric (as is the trivial case of no shear at all). Similarly, the nongeneric case of real eigenvalues of double multiplicity is more detailed, yielding either $\tilde{\mathbf{a}}$ or $\tilde{\mathbf{c}}$ moving towards a fixed direction that is a linear combination of the pair of eigenvectors.

\section{LOWEST-ORDER CORRECTION TO THE LIMITING MODEL}

The above model of Hecht et al. [23] may not capture all qualitative asymptotic behaviors of $\hat{\mathbf{a}}$ or $\hat{\mathbf{c}}$ because of the absence of effects from nonzero $r_{1}$ and $r_{2}$. In practice, e.g., for Kalliroscope particles, $r_{1}=0.2$ may not be negligible compared with 1 (even though it appears as an $r_{1}^{2}$ contribution). In the present contribution, we study the lowest-order correction in terms of $r_{1}$.

Instead of letting $r_{1}$ and $r_{2}$ both go to zero, we let $r_{2} \rightarrow 0$, and denote $\epsilon=2 r_{1}^{2} /\left(1+r_{1}^{2}\right)$ in Eq. (4), obtaining the perturbed model:

$$
\begin{gathered}
\frac{d}{d t} \hat{\mathbf{a}}=G_{21} \hat{\mathbf{b}}+G_{31} \hat{\mathbf{c}}-\epsilon E_{21} \hat{\mathbf{b}}, \\
\frac{d}{d t} \hat{\mathbf{c}}=-G_{31} \hat{\mathbf{a}}-G_{32} \hat{\mathbf{b}} .
\end{gathered}
$$

Again applying Bretherton's idea of removing the unitlength constraint on $\hat{\mathbf{c}}$, the long-time orientation of $\hat{\mathbf{c}}$ will be parallel to $\tilde{\mathbf{c}}$, with $\tilde{\mathbf{c}}$ obeying

$$
\frac{d}{d t} \tilde{\mathbf{c}}=-\mathbf{G}^{T} \cdot \tilde{\mathbf{c}} .
$$

While this consideration cannot be directly applied to â due to the small perturbation term $\epsilon E_{21} \hat{\mathbf{b}}$, Eq. (6) can still be written equivalently as:

$$
\frac{d}{d t} \hat{\mathbf{a}}=\mathbf{G} \cdot \hat{\mathbf{a}}-G_{11} \hat{\mathbf{a}}-\epsilon E_{12} \hat{\mathbf{b}} .
$$

Although the orientation of $\hat{\mathbf{a}}$ is perturbed from the eigenvector of $\mathbf{G}$, the eigenvalue analysis is still appropriate for the long-time orientation of $\hat{\mathbf{c}}$. Hence there are still three generic cases to be considered:

(i) $\mathbf{G}$ has three distinct real eigenvalues: $\tilde{\mathbf{c}}$ will align with the eigenvector of $\mathbf{G}^{T}$ (or the left eigenvector of $\mathbf{G}$ ) corresponding to its most negative eigenvalue.

(ii) $\mathbf{G}$ has a negative real eigenvalue and a pair of complex conjugate eigenvalues: $\tilde{\mathbf{c}}$ aligns with the eigenvector of $\mathbf{G}^{T}$ (or the left eigenvector of $\mathbf{G}$ ) corresponding to the negative eigenvalue.

(iii) $\mathbf{G}$ has a positive real eigenvalue and a pair of complex conjugate eigenvalues: $\tilde{\mathbf{c}}$ will rotate in the plane spanned by the two eigenvectors of $\mathbf{G}^{T}$ (or the two left eigenvectors of $\mathbf{G}$ ) corresponding to the pair of complex conjugate eigenvalues.

However, as we will show in the following sections, the long-time behavior of $\hat{\mathbf{a}}$ in the above three cases will be quantitatively different (and in the third case, qualitatively different) from the unperturbed case studied by Hecht et al. 


\section{CASE 1: THREE DISTINCT REAL EIGENVALUES}

For notational convenience, let $\hat{\mathbf{c}}_{0}$ be the unit-length eigenvector of $\mathbf{G}^{T}$ corresponding to the most negative eigenvalue. After setting $\epsilon \rightarrow 0$, the zeroth-order approximation of Eq. (9) is recovered:

$$
\frac{d}{d t} \hat{\mathbf{a}}_{0}=\mathbf{G} \cdot \hat{\mathbf{a}}_{0}-G_{11} \hat{\mathbf{a}}_{0} .
$$

Once again noting the effect of the unit-length constraint on $\hat{\mathbf{a}}_{0}$, it is now clear that $\hat{\mathbf{a}}_{0}$ will become aligned at long times with the eigenvector of $\mathbf{G}$ corresponding to the most positive eigenvalue, and we seek to identify the perturbation to the orientation of $\hat{\mathbf{a}}_{0}$.

Writing $\hat{\mathbf{a}}=\hat{\mathbf{a}}_{0}+\epsilon \mathbf{a}_{1}$ and $\hat{\mathbf{b}}=\hat{\mathbf{b}}_{0}+\epsilon \mathbf{b}_{1}$ with $\hat{\mathbf{b}}_{0}=\hat{\mathbf{c}}_{0} \times$ $\hat{\mathbf{a}}_{0}$, substituting into Eq. (9), and removing terms in the direction of $\hat{\mathbf{a}}_{0}$ (as such terms do not perturb the orientation), the first-order (in $\epsilon$ ) approximation of Eq. (9) yields

$$
\frac{d}{d t} \mathbf{a}_{1}=\left(\mathbf{G}-G_{11} \mathbf{I}\right) \mathbf{a}_{1}-E_{12} \hat{\mathbf{b}}_{0} .
$$

When $\epsilon$ is small enough we expect â to be slightly perturbed from $\hat{\mathbf{a}}_{0}$ and hence $\mathbf{a}_{1}$ will eventually align to a fixed direction. So at its steady state, we have $\frac{d}{d t} \mathbf{a}_{1}=0$ and $\mathbf{a}_{1}$ satisfies the equation:

$$
\left(\mathbf{G}-G_{11} \mathbf{I}\right) \mathbf{a}_{1}=E_{12} \hat{\mathbf{b}}_{0} .
$$

Since the desirable $\mathbf{a}_{1}$ here is in the space orthogonal to $\hat{\mathbf{a}}_{0}$, we substitute $\mathbf{a}_{1}=y \hat{\mathbf{b}}_{0}+z \hat{\mathbf{c}}_{0}$ into Eq. (12), left multiply both sides by $\hat{\mathbf{b}}_{0}^{T}$ and $\hat{\mathbf{c}}_{0}^{T}$ respectively, and obtain the solution

$$
\mathbf{a}_{1}=\frac{E_{12}}{\hat{\mathbf{b}}_{0}^{T}\left(\mathbf{G}-G_{11} \mathbf{I}\right) \hat{\mathbf{b}}_{0}} \hat{\mathbf{b}}_{0} .
$$

In a prescribed flow, the above solution breaks down when $\epsilon$ is large enough, as â may no longer be fixed and instead rotates in the plane orthogonal to $\hat{\mathbf{c}}$, as is intuitively understandable by reconsidering Eq. (9): When the perturbation is large, the right-hand side is dominated by $\epsilon E_{12} \hat{\mathbf{b}}$, constrained to be orthogonal to $\hat{\mathbf{a}}$ and in the plane perpendicular to $\hat{\mathbf{c}}$, so it drags â into the orbit of rotation. In other words, which behavior the particle shows depends on the amount of vorticity relative to a component of the strain rate multiplied by $\epsilon$, and thus depends on the combined effect of the flow gradient and the particle's aspect ratio. However, we focus here on the different behaviors of the particle for different $\epsilon$ in a given flow field. Numerical verifications are shown below.

We randomly generate velocity gradient matrices $\mathbf{G}$ that have three distinct real eigenvalues, and for each $\mathbf{G}$ find numerical solutions to the full system Eq. (4) for ellipsoids with different aspect ratios. We then compare our asymptotic approximation $\hat{\mathbf{a}}=\hat{\mathbf{a}}_{0}+\epsilon \mathbf{a}_{1}$ [Eq. (10) and Eq. (13)] to the actual solution and plot the absolute error (measured in $\infty$ norm) against the magnitude of $|\epsilon|$ in Fig. 1. For each given flow gradient $\mathbf{G}$, as expected the two distinct types of behaviors of $\hat{\mathbf{a}}$ as $\epsilon$ increases are reflected in the figure. When $\epsilon$ is small, the actual $\hat{\mathbf{a}}$ is well approximated by our asymptotic solution with a difference smaller than 0.1 . As $\epsilon$ crosses past a critical point (with specific details set by properties of $\mathbf{G}$ ), there is a transition in the behavior of â after which our underlying assumption of an attracting stable orientation is no longer valid.

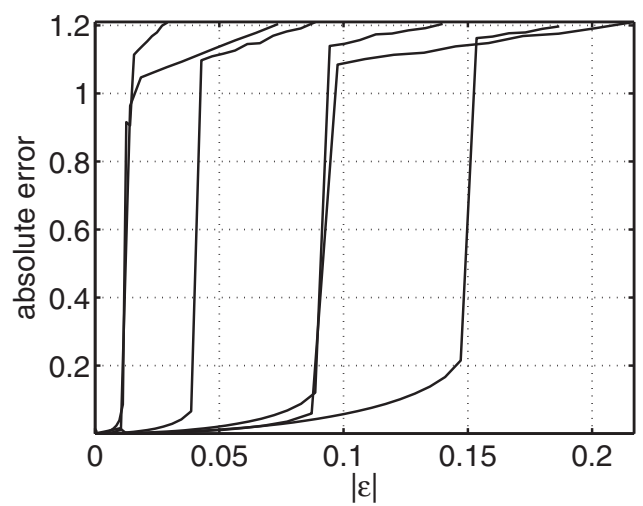

FIG. 1. Absolute error between our asymptotic solution and the numerical solution of the full system Eq. (4) for ellipsoids with different aspect ratio $r_{1}$ (and hence different $\epsilon$ ), and fixed $r_{2}=0.002$. Different curves correspond to different gradient velocity tensors $\mathbf{G}$ that satisfy Case 1 .

The numerical solution shows that $\mathbf{a}$ is rotating in the plane perpendicular to the fixed $\hat{\mathbf{c}}$ in these situations (Fig. 2).

\section{CASE 2: A NEGATIVE REAL EIGENVALUE AND COMPLEX CONJUGATE PAIR}

In the absence of the perturbation $E_{12} \hat{\mathbf{b}}, \hat{\mathbf{a}}_{0}$ rotates in the plane spanned by the eigenvectors of $\mathbf{G}$ that correspond to the pair of complex conjugate eigenvalues (i.e., the plane perpendicular to $\hat{\mathbf{c}}_{0}$ ). Recognizing that this perturbation is in the direction of $\hat{\mathbf{b}}$, which is always orthogonal to $\hat{\mathbf{a}}$ and $\hat{\mathbf{c}}_{0}$, the long-time effect of a small perturbation only influences the detailed motion of the rotation of $\mathbf{a}$ in the same plane, as shown in Fig. 3, which plots the components of the actual $\hat{\mathbf{a}}$ found numerically and the solution to the zeroth-order equation $\frac{d}{d t} \hat{\mathbf{a}}_{0}=\mathbf{G} \cdot \hat{\mathbf{a}}_{0}-G_{11} \hat{\mathbf{a}}_{0}$ as a reference. It ensures that the two solutions are rotating in the same orbit but they are out of phase.

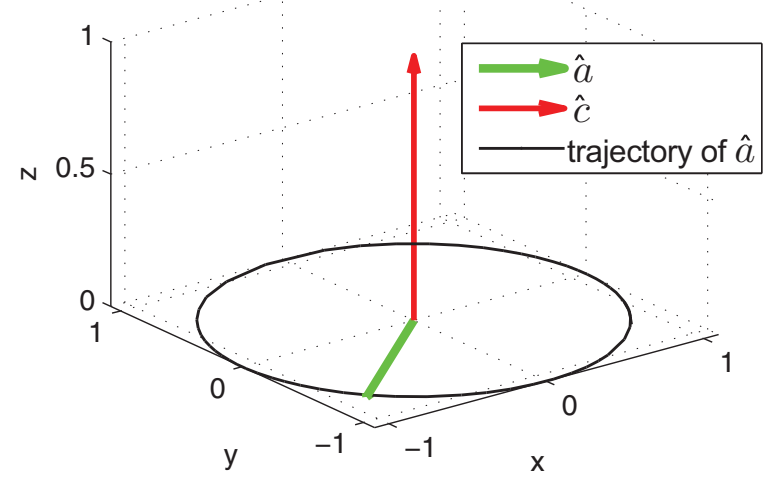

FIG. 2. (Color online) Trajectories of the vectors $\hat{\mathbf{a}}$ and $\hat{\mathbf{c}}$ of an ellipsoid with aspect ratio $r_{1}=0.5(\epsilon=0.4)$ in a prescribed velocity field. The vector $\hat{\mathbf{c}}$ (red) is fixed and parallel to the $\mathrm{z}$ axis. The vector $\mathbf{a}$ (green) lies in the plane perpendicular to $\hat{\mathbf{c}}$ (the $x-y$ plane) and rotates in the plane under the strong influence of the perturbation term $\epsilon E_{12} \hat{\mathbf{b}}$, in contrast to the $\epsilon \ll 1$ behavior of Case 1 . 

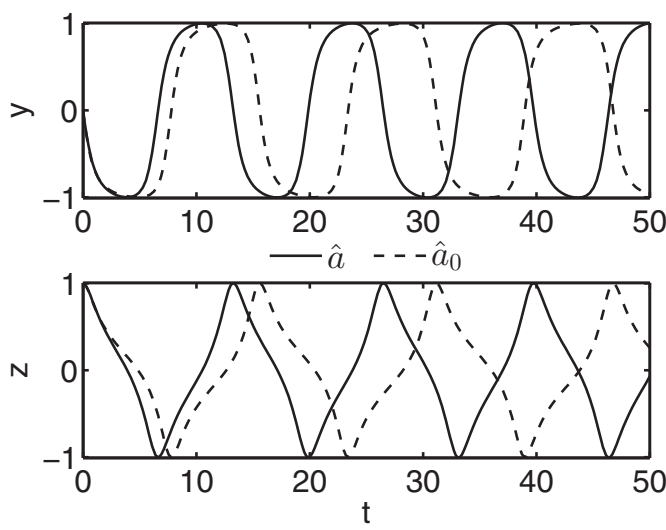

FIG. 3. Components of $\hat{\mathbf{a}}$ in the numerical solution of the full system Eq. (4) and of $\hat{\mathbf{a}}_{0}$ in the solution to the zeroth-order equation $\frac{d}{d t} \hat{\mathbf{a}}_{0}=\mathbf{G} \cdot \hat{\mathbf{a}}_{0}-G_{11} \hat{\mathbf{a}}_{0}$, as an example in Case 2. The ellipsoid in this example has aspect ratios $r_{1}=0.3(\epsilon=0.17)$ and $r_{2}=0.002$. Because $\hat{\mathbf{c}}_{0}$ is aligned to $(1,0,0)$ here, the $x$ coordinates of $\hat{\mathbf{a}}$ and $\hat{\mathbf{a}}_{0}$ are identically 0 .

\section{CASE 3: A POSITIVE REAL EIGENVALUE AND A COMPLEX CONJUGATE PAIR}

This is the most interesting case at this level of perturbation, with $\hat{\mathbf{c}}$ rotating in the plane spanned by the two eigenvectors of $\mathbf{G}^{T}$ corresponding to the pair of complex conjugate eigenvalues. In order to elucidate the full orientation of the scalene ellipsoid, it is convenient to work in a new fixed reference frame in which $\mathbf{G}$ will take a simpler form: Choosing the unit-length eigenvector that corresponds to the real eigenvalue of $\mathbf{G}$ as the new $x$ axis, and picking any other two orthonormal vectors to form a new reference frame, in the new laboratory frame $\mathbf{G}$ takes the form

$$
\left[\begin{array}{lll}
\lambda & * & * \\
0 & * & * \\
0 & * & *
\end{array}\right]
$$

where $\lambda$ is the real eigenvalue of $\mathbf{G}$. In this reference frame, Eq. (6) simplifies to

$$
\frac{d}{d t} \hat{\mathbf{a}}=\mathbf{G} \cdot \hat{\mathbf{a}}-\lambda \hat{\mathbf{a}}-\frac{\epsilon}{2} G_{12} \hat{\mathbf{b}} .
$$

Analogous to the development of Eqs. (10)-(11), substituting in $\hat{\mathbf{a}}=\hat{\mathbf{a}}_{0}+\epsilon \mathbf{a}_{1}$ gives the zeroth-order approximation and the first-order correction of $\hat{\mathbf{a}}$ :

$$
\begin{gathered}
O(1): \frac{d}{d t} \hat{\mathbf{a}}_{0}=\mathbf{G} \cdot \hat{\mathbf{a}}_{0}-\lambda \hat{\mathbf{a}}_{0}, \\
O(\epsilon): \frac{d}{d t} \mathbf{a}_{1}=(\mathbf{G}-\lambda \mathbf{I}) \mathbf{a}_{1}-\left(\mathbf{v} \cdot \hat{\mathbf{b}}_{0}\right) \hat{\mathbf{b}}_{0},
\end{gathered}
$$

where $\mathbf{v}=\hat{\mathbf{a}}_{0}^{T} \cdot \mathbf{G} / 2$ and $\hat{\mathbf{b}}_{0}=\hat{\mathbf{c}} \times \hat{\mathbf{a}}_{0}$. In the specially selected reference frame, $\hat{\mathbf{a}}_{0}$ is simply $(1,0,0)^{T}$ and $\hat{\mathbf{b}}_{0}=\hat{\mathbf{c}} \times \hat{\mathbf{a}}_{0}$ is not fixed because $\hat{\mathbf{c}}$ rotates. Again, we are only interested in the projection of $\mathbf{a}_{1}$ onto the plane orthogonal to $\hat{\mathbf{a}}_{0}$ (i.e., the $y-z$ plane in this case), while $\mathbf{a}_{1}$ must also be orthogonal to $\hat{\mathbf{c}}$ by definition. Therefore, $\mathbf{a}_{1}$ must be some (possibly negative) multiple of $\hat{\mathbf{b}}_{0}$, i.e., $\hat{\mathbf{b}}_{0}= \pm \mathbf{a}_{1} /\left\|\mathbf{a}_{1}\right\|$. Substituting into the above equation and denoting $(\mathbf{G}-\lambda \mathbf{I})$ by $\tilde{\mathbf{G}}$, Eq. (16) becomes

$$
\frac{d}{d t} \mathbf{a}_{1}=\tilde{\mathbf{G}} \cdot \mathbf{a}_{1}-\left(\mathbf{v} \cdot \mathbf{a}_{1}\right) \frac{\mathbf{a}_{1}}{\left\|\mathbf{a}_{1}\right\|^{2}}
$$

For simplicity - and to develop intuition-we analytically consider the special case of the above where $\tilde{\mathbf{G}}$ is a rotation matrix acting around the $x$ axis (that is, rotating the $y-z$ plane). Then, for general $\tilde{\mathbf{G}}$, our numerical results show similar behaviors.

Assume $\tilde{\mathbf{G}}=\mathbf{G}-\lambda \mathbf{I}$ is a rotation matrix on the $y-z$ plane with angle $\theta$,

$$
\tilde{\mathbf{G}}=\left[\begin{array}{ccc}
0 & * & * \\
0 & \cos \theta & -\sin \theta \\
0 & \sin \theta & \cos \theta
\end{array}\right] .
$$

We note the requirement that $\mathbf{G}$ has trace 0 gives that $\tilde{\mathbf{G}}=$ $\mathbf{G}-\lambda \mathbf{I}$ has trace $-3 \lambda$ and $2 \cos \theta=-3 \lambda<0$, requiring the rotation angle $\theta$ be larger than 90 degrees. Letting $[\alpha(t), \rho(t)]$ denote the polar coordinates of $\mathbf{a}_{1}$ on the $y-z$ plane and $\beta$ be the angle between $\mathbf{v}$ and positive $x$ axis, Eq. (17) when restricted on the $y-z$ plane can be transformed into polar coordinates $[\alpha(t), \rho(t)]$ :

$$
\begin{gathered}
\frac{d}{d t} \alpha=\sin \theta, \\
\frac{d}{d t} \rho=\rho \cos \theta-|\mathbf{v}| \cos (\alpha-\beta),
\end{gathered}
$$

with solutions

$$
\begin{gathered}
\alpha(t)=\alpha_{0}+t \sin \theta, \\
\rho(t)=e^{t \cos \theta}\left(\rho_{0}-|\mathbf{v}| \cos \left(\alpha_{0}-\beta+\theta\right)\right) \\
+|\mathbf{v}| \cos \left(\theta-\beta+\alpha_{0}+t \sin \theta\right),
\end{gathered}
$$

where $\left(\alpha_{0}, \rho_{0}\right)$ is the initial position. Therefore, $\mathbf{a}_{1}$ is moving in an orbit on the $y-z$ plane with time-dependent radius in its attracting state regardless of its initial condition. Specifically,

(i) $e^{t \cos \theta} \rightarrow 0$ as $t \rightarrow \infty$ since $\cos \theta<0$, so in its attracting state $\mathbf{a}_{1}$ is rotating in the orbit on the $y-z$ plane: $\rho(t)=|\mathbf{v}| \cos [\theta-\beta+\alpha(t)]$, which is independent of the initial position $\left(\alpha_{0}, \rho_{0}\right)$.

(ii) The point $t^{*}$ at which $\rho\left(t^{*}\right)=0$ is a singular point. When $\rho\left(t^{*}\right)=|\mathbf{v}| \cos \left(\alpha\left(t^{*}\right)+\theta-\beta\right)=0, \alpha\left(t^{*}\right)=\beta-\theta+$ $\frac{\pi}{2}+k \pi$. Hence when $\mathbf{a}_{1}$ passes the origin, there will be a jump of $\pi$ between the entrance angle and the exit angle.

Numerical results confirm the orbit of $\mathbf{a}_{1}$ obtained from our first-order correction. Figure 4 shows the projection on the $y-z$ plane of â computed numerically from the full system Eq. (4), compared to our prediction of the orbit of $\mathbf{a}_{1}$ on the $y-z$ plane [Eqs. (20)-(21)] for a selected $\mathbf{G}$ with desired eigenvalues and with its projection $\tilde{\mathbf{G}}$ as a rotation matrix.

For general $\tilde{\mathbf{G}}$, which is not necessarily a rotation, we solve Eq. (17) numerically and find the same type of orbit for $\mathbf{a}_{1}$. Figure 5 compares the projection on the $y-z$ plane of the actual â computed numerically from the full system Eq. (4) with the $\mathbf{a}_{1}$ obtained numerically from our first-order correction [Eq. (17)], and it substantiates the conclusion that 


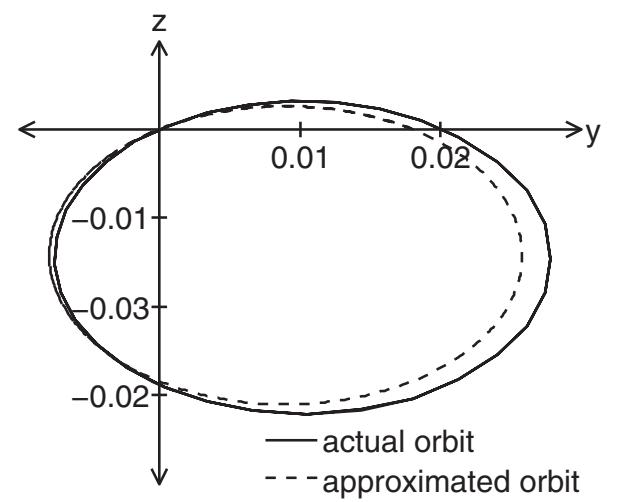

FIG. 4. Orbit of â on the $y-z$ plane computed numerically from the full system Eq. (4) and the analytical solution from our first-order approximation [Eqs. (20)-(21)] in Case 3 for $\tilde{\mathbf{G}}$ as a rotation matrix with rotation angle $158^{\circ}$. The ellipsoid has aspect ratio $r_{1}=0.2$ and $r_{2}=0.002$ (similar to Kalliroscope particles).

the first-order model well approximates the full dynamics and the wobbling behavior of $\mathbf{a}$ is general in this context.

\section{EFFECTS OF NONZERO SECOND ASPECT RATIO}

Our perturbation analysis above has only considered the lowest-order correction to the base state-that is, including the lowest-order effects of $r_{1}$ under the assumption that $r_{1} \gg r_{2}$. Although real particles have nonzero $r_{2}$, this approximation would seem to be appropriate for Kalliroscope particles, where $r_{1}=0.2$ and $r_{2} \approx 2 \times 10^{-3}$ [10]). Indeed, as we have seen above, our asymptotic results-which effectively treat $r_{2}$ as zero-agree well with the numerical solutions to the full system Eq. (4) with $r_{2}=2 \times 10^{-3}$.

Noting that $r_{1}$ and $r_{2}$ enter the full equations as squares of their respective values, it is reasonable that the nonzero aspect ratio $r_{2}$ of these particles at such small values will not change the qualitative behavior predicted by the perturbation analysis for nonzero $r_{1}$. The lowest-order appearance of $r_{2}$ in the expansion of the full equations is in the $E_{32} \hat{\mathbf{b}}$ term

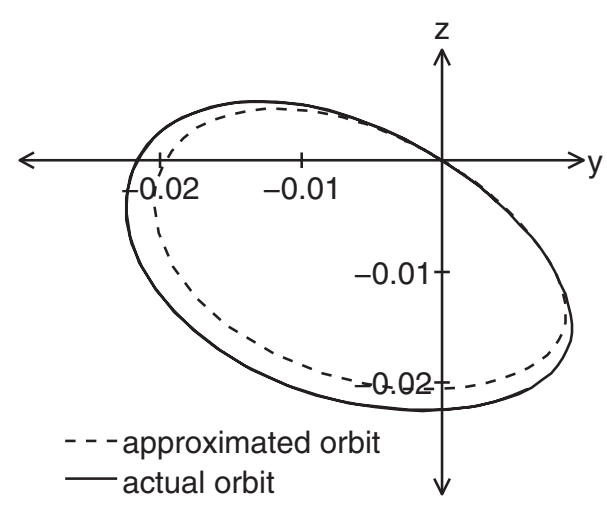

FIG. 5. Orbits on the $y-z$ plane of â computed numerically from the full system Eq. (4) and from the first order model [Eq. (17)] for a G with one positive real eigenvalue and a negative real-part complexconjugate pair (Case 3). The ellipsoid has aspect ratio $r_{1}=0.2$ and $r_{2}=0.002$ (similar to Kalliroscope particles). in the $\frac{d}{d t} \hat{\mathbf{c}}$ equation, as a multiplying factor $\delta \equiv 2 r_{2}^{2} /\left(r_{1}^{2}+\right.$ $\left.r_{2}^{2}\right) \approx 3 \times 10^{-4}$. In contrast, the first-order-in- $\epsilon$ correction $\left[\epsilon=2 r_{1}^{2} /\left(1+r_{1}^{2}\right) \approx 0.08\right]$ considered in the present work has already neglected $O\left(\epsilon^{2}\right)$ terms, with $\epsilon^{2} \approx 6 \times 10^{-3}$. That said, the $O(\delta)$ correction is qualitatively different in that it affects the dynamics of the short axis $\hat{\mathbf{c}}$. Because of this impact, the simple eigenvector-driven analysis performed here no longer immediately applies to all cases. It is perhaps interesting to note that the effect of this small additional correction will only be to slightly modify the orbit of $\hat{\mathbf{c}}$ in the $\hat{\mathbf{b}}$ direction. In Case 1 , we then continue to expect fixed orientations for sufficiently small aspect ratios. In Cases 2 and 3, however, the perturbation of both $\hat{\mathbf{a}}$ and $\hat{\mathbf{c}}$ will cause simultaneous wobbling of the fixed-at-zeroth-order axis and push the rotating axis out of the plane corresponding to the complex conjugate eigenvalues.

To further understand the domain where it may be appropriate to ignore the effects of nonzero $r_{2}$, we close with additional numerical experiments, varying $r_{2}$. As $r_{2}$ increases, the dynamics of the $\hat{\mathbf{c}}$ axis gradually deviate from its zeroth-order model, which renders our first-order-in- $\epsilon$ model invalid when $r_{2}$ is large. A special case is when $r_{2}=r_{1}$ (axisymmetric); as noted by Bretherton [24], the axis â of an axisymmetric particle is either fixed or rotating in a plane, and thus the wobbling behavior found for a triaxial particle must disappear. To demonstrate how $r_{2}$ affects the wobbling motion, we numerically solve the full system Eq. (4) in a prescribed flow in Case 3 with increasing $r_{2}$ values from 0 to 0.2 , and plot the stationary orbits of â corresponding to different $r_{2}$ 's, compared to the first-order-in- $\epsilon$ results $\left(r_{2}=0\right)$. The numerical results for $r_{2}=0,0.002$, and 0.02 plotted in Fig. 6 are effectively indistinguishable from one another. On the order of 0.1 , the finite value of $r_{2}$ does not change the qualitative behavior of the particle and the wobbling motion of $\hat{\mathbf{a}}$ is still observable, but the orbit has shifted significantly. When $r_{2}=0.2=r_{1}$ we can see that the wobbling motion vanishes as â aligns to a fixed direction.

For completeness, we note some of the similarities and differences of these trajectories with those observed in the

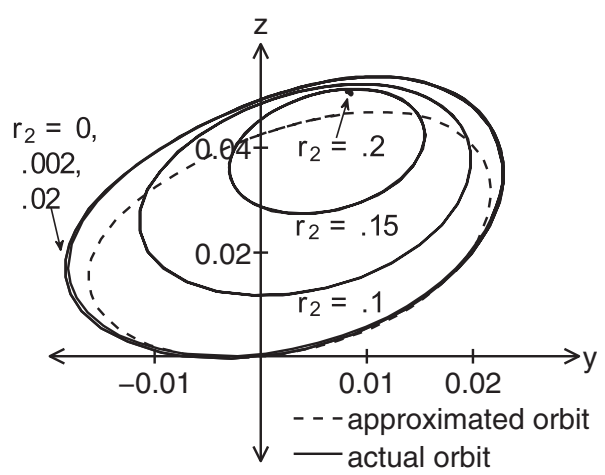

FIG. 6. Orbits on the $y-z$ plane of â computed numerically from the full system Eq. (4) in a prescribe flow in Case 3 with increasing $r_{2}$ values from 0 to $0.2 . r_{1}$ is fixed at 0.2 . The orbit of $\mathbf{a}$ obtained from the first-order model [Eq. (17)] is also shown for comparison. The numerical results for $r_{2}=0,0.002$, and 0.02 are indistinguishable. When $r_{2}=0.2=r_{1}$ the orbit shrinks to a point and $\hat{a}$ aligns to a fixed direction. 
numerical solution of the equations by Gauthier et al. [21] The periodic trajectories of the end of the longest axis (i.e., a) that they presented were obtained with fixed $r_{2}=0.1$ for $0.1 \leqslant r_{1} \leqslant 1$, over which the extremity of the longest axis precesses with the amplitude of precession increasing as a long prolate spheroid deforms to a thin oblate one. Despite some of the qualitative similarities of the motions involved, because of the large $r_{2}=0.1$ second aspect ratio there, the lowest-order corrections considered here are not expected to be quantitatively accurate (see, for example, the $r_{2}=.1$ trajectory in Fig. 6).

\section{CONCLUSION}

This paper investigates the orientational motion of nonaxisymmetric (scalene or triaxial) ellipsoids immersed in a locally linear 3D incompressible flow, focusing on the enumeration of the different qualitative behaviors obtained by consideration of the extreme aspect ratios typical of rheoscopic particles, where the long-time orientation can be well approximated by models that are at low order in the aspect ratios. It is important to recognize that accurate calculation of particle motions requires numerical solution of the underlying differential equations (e.g., as in Gauthier et al. [21]) for anything beyond the most extreme aspect ratios. The perturbative analysis considered here addresses the possible qualitative features of the long-time behavior of particle orientations in terms of the types of eigenvalues of the gradient velocity tensor, reviewing previous results about the limiting base model and exploring results obtained in the extension to the lowest-order correction in the larger of the two aspect ratios. In the case of a single positive real eigenvalue with a (negative real part) complex conjugate pair, we demonstrate that small perturbative effects lead to a qualitatively different behavior; in particular, the lowest-order correction describes a long-time periodic wobbling motion of the particle.

\section{ACKNOWLEDGMENT}

This work was partially supported by NSF Grant No. DMS0645369, ARO Grant No. 12-60317-MS, and AFOSR Grant No. FA9550-12-1-0178.
[1] G. B. Jeffery, Proc. R. Soc. London, Ser. A 102, 161 (1922).

[2] G. I. Taylor, Proc. R. Soc. London, Ser. A 103, 58 (1923).

[3] R. C. Binder, J. Appl. Phys. 10, 711 (1939).

[4] P. G. Saffman, J. Fluid. Mech. 1, 540 (1956).

[5] J. Feng and D. D. Joseph, J. Fluid. Mech. 303, 83 (1995).

[6] S. G. Mason and R. S. J. Manley, Proc. R. Soc. London, Ser. A 238, 117 (1956)

[7] L. G. Leal and E. J. Hinch, J. Fluid. Mech. Digital Archive 46, 685 (1971)

[8] E. J. Hinch and L. G. Leal, J. Fluid. Mech. Digital Archive 52, 683 (1972).

[9] L. G. Leal and E. J. Hinch, Rheologica Acta 12, 127 (1973).

[10] P. Matisse and M. Gorman, Phys. Fluids 27, 759 (1984).

[11] S. T. Thoroddsen and J. M. Bauer, Phys. Fluids 11, 1702 (1999).

[12] W. Salalha and E. Zussman, Phys. Fluids 17, 063301 (2005).

[13] E. Katz, A. L. Yarin, W. Salalha, and E. Zussman, J. Appl. Phys. 100, 034313 (2006).

[14] J. R. T. Seddon and T. Mullin, J. Fluid. Mech. 583, 123 (2007).
[15] V. Bezuglyy, B. Mehlig, and M. Wilkinson, Europhys. Lett. 89, 34003 (2010).

[16] S. Goto, S. Kida, and S. Fujiwara, J. Fluid. Mech. 683, 417 (2011).

[17] P. J. Gierszewski and C. E. Chaffey, Can. J. Phys. 56, 6 (1978).

[18] E. J. Hinch and L. G. Leal, J. Fluid. Mech. 92, 591 (1979).

[19] A. L. Yarin, O. Gottlieb, and I. V. Roisman, J. Fluid. Mech. 340, 83 (1997).

[20] M. Wilkinson, V. Bezuglyy, and B. Mehlig, J. Fluid. Mech. 667, 158 (2011).

[21] G. Gauthier, P. Gondret, and M. Rabaud, Phys. Fluids 10, 2147 (1998).

[22] W. L. Barth and C. A. Burns, IEEE Trans. Visual. Comput. Graph. 13, 1751 (2007).

[23] F. Hecht, P. J. Mucha, and G. Turk, IEEE Trans. Visual. Comput. Graph. 16, 147 (2010).

[24] F. P. Bretherton, J. Fluid. Mech. 14, 284 (1962). 